\title{
L'artisan, son comptable et sa retraite
}

Étude d'un profil d'indépendants établis

Contributing to Pension Schemes and (Still) Investing in Brick. Accommodations between Building Craftsmen and their Accountants

\section{Marine Guichard et Marielle Poussou-Plesse}

\section{(2) OpenEdition \\ Journals}

Édition électronique

URL : http://journals.openedition.org/travailemploi/7424

DOI : 10.4000/travailemploi.7424

ISSN : $1775-416 \mathrm{X}$

Éditeur

DARES - Ministère du Travail

Édition imprimée

Date de publication : 1 janvier 2017

Pagination : 43-72

ISSN : 0224-4365

Référence électronique

Marine Guichard et Marielle Poussou-Plesse, «L'artisan, son comptable et sa retraite », Travail et Emploi [En ligne], 149 I janvier-mars 2017, mis en ligne le 11 juillet 2019, consulté le 07 septembre 2019. URL : http://journals.openedition.org/travailemploi/7424; DOI : 10.4000/travailemploi.7424 


\title{
L'artisan, son comptable et sa retraite Étude d'un profil d'indépendants établis
}

\author{
Marine Guichard ${ }^{*}$, Marielle Poussou-Plesse ${ }^{* *}$
}

\begin{abstract}
En complément des constats statistiques de la recherche sur l'accumulation patrimoniale chez les indépendants, l'article vise à contribuer à une compréhension des choix faits par les artisans pour préparer leur retraite. Il prend au sérieux la persistance d'une conception professionnelle de la retraite, fondée sur un souci d'autoprotection de ses vieux jours, qui semble expliquer un niveau de détention patrimoniale des ménages indépendants très supérieur à celui des ménages salariés. Étayée par une enquête qualitative, à la fois circonscrite géographiquement et centrée sur un profil d' artisans du bâtiment établis, l'analyse propose de rendre compte de deux mécanismes observés de «mise au second plan » de la pension versée par le régime social des indépendants (RSI). Cette mise au second plan montre en creux l'importance subjective et objective pour les enquêtés de constituer leur propre retraite. La relation de ces indépendants à leur cabinet comptable sert de fil directeur. L'étude de cette médiation sociale permet en effet de dégager deux dimensions distinctes d'une conception professionnelle de la retraite : l'une attachée à une socialisation au statut d'indépendant par le réalisme comptable ; l'autre liée aux normes d'accomplissement dans le métier et qui autorise les artisans à s'affranchir de ce réalisme en investissant dans la pierre.
\end{abstract}

L a littérature exploitant l'enquête Patrimoine de l'Institut national de la statistique et des études économiques (Insee) montre à quel point l'accumulation patrimoniale continue de distinguer les ménages indépendants des ménages salariés. En plus d'établir la très nette supériorité des montants moyens de patrimoine total des premiers par rapport aux seconds (INSEE, 2015), elle s'est attachée à décrire les différences internes au groupe des indépendants : différences en termes de montants du patrimoine total,

\footnotetext{
* Laboratoire lorrain de sciences sociales (2L2S, EA 3478, Metz), université de Lorraine ; marine.guichard@univlorraine.fr.

** Centre Georges Chevrier, université de Bourgogne - Franche-Comté (CGC, UMR 7366, Dijon); marielle. poussou@u-bourgogne.fr.
} 
de composition (actifs professionnels, actifs privés) et d'évolution de ces montants et de leur composition avec le cycle de vie, notamment avec le passage à la retraite (BlANPAIN, 2000 ; Bessière et al., 2011 ; Bourdieu et al., 2014). Dans ces travaux, les déterminants des stratégies d'accumulation patrimoniale, des stratégies de conversion des actifs (actifs professionnels versus actifs privés) et des stratégies de transmission sont systématiquement testés par des modèles économétriques. Cette approche statistique débouche sur le constat majeur que le patrimoine professionnel, composante spécifique du patrimoine des ménages non salariés, ne suffit pas à rendre compte de la supériorité de leur patrimoine total, conduisant ainsi à l'interrogation suivante :

«Au-delà de l'importance de sa composante professionnelle, en quoi le patrimoine des indépendants se distingue-t-il de celui des autres ménages ? Les ménages d'indépendants ont-ils des comportements patrimoniaux spécifiquement liés à leur statut, par exemple au fait d'être davantage que les salariés exposés à de fortes fluctuations de leurs revenus d'activité ?»

(INSEE, 2015, p. 75)

L'interprétation avancée est régulièrement la même : le fait que le patrimoine privé des ménages indépendants soit près de deux fois supérieur $(84 \%)$ en moyenne en 2010 à celui des ménages salariés, pourrait s'expliquer par

« une propension plus forte des ménages d'indépendants à se constituer une épargne dite de précaution pour faire face aux fluctuations plus fortes du revenu, liées à des facteurs conjoncturels (cycles d'activité) ou structurels (plus faibles prestations des régimes de retraite) ».

(Ibid., p. 80)

C'est cette logique de «précaution » avancée par l'approche statistique sur le mode de l'explication plausible ${ }^{1}$ que le présent article vise à étayer et à préciser par les apports d'une enquête qualitative. Centrée sur la constitution de sa retraite chez un profil d'indépendant établi (encadré 1), elle montre l'importance, objective et subjective, de « se faire sa retraite ». Bien qu'elle s'inscrive dans la lignée du constat général d'une logique d'accumulation patrimoniale spécifique aux non-salariés, cette importance ne manque pas d'interroger. L'intérêt scientifique pour la question patrimoniale chez les indépendants s'est affirmé avec le processus institutionnel, continu depuis 1973, d'alignement de la protection sociale des indépendants sur le régime général, dans lequel

\footnotetext{
1. Le dispositif de l'enquête Patrimoine ne permet pas méthodologiquement de tester cette hypothèse. En effet, et c'est une limite générale dont les auteurs ont conscience, l'enquête ne repose pas sur des données de panel, ce qui conduit à mener un raisonnement en termes de cycle de vie sur des ménages différents, observés en coupe à des âges distincts. À cette limite générale s'ajoutent les difficultés d'une estimation des taux d'épargne, estimation qu'implique le test de l'hypothèse, la difficulté principale tenant au caractère peu fiable des données de revenus déclarés. Enfin, jusqu'à sa dernière édition (2015), l'enquête ne permettait aucunement de sonder la représentation d'une « faiblesse » des prestations de retraite et sa place dans les motivations à accumuler du patrimoine. Le questionnaire interroge désormais sur les motivations à épargner. Son exploitation montre une nette surreprésentation du motif « préparer ses vieux jours » chez les indépendants agricoles (31\%) et non agricoles (27\%), par rapport aux cadres et aux ouvriers (moins de $17 \%$ dans les deux cas) (INSEE, 2016).
} 
s'inscrit la mise en place du Régime social des indépendants (RSI) en $2006^{2}$ (BRUNO, 2014). Logiquement, au sein de la littérature statistique, un questionnement s'est tourné plus précisément vers l'observation d'une éventuelle recomposition des revenus des indépendants retraités. Autrement dit, avec l'amélioration des droits à pension des indépendants visée notamment par le processus d'alignement, les ressources patrimoniales tendraient-elles à relativement décliner au regard des ressources du « salaire socialisé » auquel s'apparente la pension par répartition ${ }^{3}$ ? Dans une publication datée, mais portant explicitement ce questionnement, les résultats de l'exploitation de l'ancienne enquête Patrimoine concluent aux « limites du processus d'harmonisation » sur les comportements des travailleurs indépendants en matière de préparation de leur retraite (DuBOISLAMBERT, 1997). Plus généralement, « la faiblesse relative, réelle ou ressentie, de leur protection sociale ${ }^{4} »$ continue d'être invoquée dans les raisonnements statistiques sur l'accumulation patrimoniale comme explication du fait que les ménages d'indépendants n'accordent qu' un rôle d'appoint à la pension. Le registre économétrique de démonstration n'échappe pas à un risque de circularité : l'approche microéconomique des modèles du cycle de vie tend à donner une rationalisation a posteriori des comportements sur la base des montants constatés de patrimoine et des rentes tirées de ses différentes composantes (professionnelle, immobilière, financière) $)^{5}$. Or les capacités stratégiques des acteurs que présupposent de tels modèles méritent d'être interrogées à la fois en termes de (mé)connaissance ordinaire des paramètres d'optimisation par les intéressés et en termes de médiations sociales requises pour les maîtriser.

2. La médiatisation récente du RSI a pu laisser penser au grand public que cette « Sécu des indépendants » était vraiment spécifique. Pourtant, sa mise en place a été un moment fort de l'harmonisation des régimes sociaux (jusque-là autonomes) des différentes catégories d'indépendants non agricoles, harmonisation permettant à son tour d'approfondir leur alignement avec le régime général jusqu'à poser la question, débattue au moment où ces lignes sont écrites (avril 2017) de leur totale intégration, signifiant de fait la disparition du RSI.

3. Avant la mise en œuvre du processus d'alignement en 1973, le régime autonome des artisans, créé en 1948, se présentait comme un système mixte par points : aux cotisations sociales minimales obligatoires fonctionnant en répartition s'ajoutait une dose de capitalisation via la possibilité de cotiser volontairement selon des paliers. Depuis 1973, les règles de calcul des droits et les niveaux de cotisations des artisans pour la retraite de base ont été rapprochés de ceux des salariés au point d'être considérés aujourd'hui comme leur étant identiques. Un régime de retraite complémentaire obligatoire des artisans a été créé en 1979, il est aujourd'hui géré par le RSI dans le cadre d'un régime complémentaire devenu commun avec les commerçants. Comme pour ceux des salariés (Association générale des institutions de retraite complémentaire des cadres - Association pour le régime de retraite complémentaire des salariés [Agirc-Arrco]), ce régime complémentaire fonctionne par points et jusqu'au plafond de la Sécurité sociale, les taux de cotisation sont similaires à ceux des salariés. Au-delà, les indépendants cotisent moins. Le financement de ce régime reste par ailleurs caractérisé par une originalité : il ne s'agit pas d'un système de répartition pure au sens où la constitution de réserves est au cœur de son pilotage (COR, 2009).

4. Cf. l'article fondateur de Jean-Jacques MALPOT et Nathalie Missègue (1996, p. 91).

5. Il en va ainsi de formulations, qui par ailleurs rendent compte de manière juste de ce que les économistes appellent des « faits stylisés », telles que : « à la différence des salariés, la retraite des indépendants résulte donc davantage d'une optimisation simultanée de leurs décisions d'offre de travail et d'accumulation patrimoniale sur leur cycle de vie » ou « l'effort de cotisation relève, en fait, d'une stratégie financière ex ante, c'est-à-dire d'un arbitrage endogène entre une préparation individuelle et collective de leur retraite » (DuBoIs, LAMBERT, 1997, p. 2). Pour rappel, les modèles de cycle de vie visent à rendre compte des comportements d'épargne et de désépargne au cours de la vie, lesquels seraient orientés selon le modèle fondateur de Modigliani par une volonté de maintenir stable dans le temps le niveau de consommation. Les développements ultérieurs ont intégré deux autres préoccupations : la transmission aux enfants et les imprévus (dont ceux liés aux dépenses de santé). 


\section{ENCADRÉ 1}

\section{Démarche et corpus}

Le matériau empirique est constitué de deux types d'entretiens conduits en 2015-2016.

Vingt-sept entretiens ont été menés auprès d'artisans du bâtiment ; la moitié d'entre eux étaient en fin de carrière (plus de 50 ans), et les autres venaient de liquider leur pension. Tous les corps de métiers sont représentés (une entreprise générale, quatre maçons, trois menuisiers, deux charpentiers, sept plâtriers-peintres, trois plombiers-chauffagistes, deux électriciens, deux plaquistes, un serrurier-métallier, un carreleur, un ferronnier). La constitution de ce corpus à partir des renseignements fournis par un informateur avec lequel l'une des enquêtrices a un rapport de parenté, lui donne un caractère fort de réseau local. Ces artisans sont tous implantés dans une zone rurale d'une région viticole, aujourd'hui à faible dynamique migratoire. La communauté portugaise y est la plus représentée en raison d'importantes vagues d'immigration dans les années $1970^{1}$.

Les enquêtés étaient sollicités pour évoquer leurs attentes et leurs expériences en matière de retraite (revenus post-professionnels et mode de vie) mais aussi en matière de santé au travail et ce, dans une perspective biographique. Par recoupements, l'analyse de leurs discours a dégagé un profil majoritaire (22 cas sur les 27 enquêtés) d'indépendant (bien) établi aux trois caractéristiques suivantes.

1) Ils sont installés dans le métier au minimum depuis plus de vingt ans. Leur carrière est marquée par la norme, qui préside toujours à la décision de s'établir dans l'artisanat, d'ascension sociale par rapport à la condition d'ouvrier du bâtiment, mais aussi par l'expérience d'avoir, sur la durée, surmonté les aléas à l'origine de l'irrégularité de leur trésorerie et, a fortiori, d'avoir évité le dépôt de bilan qu'ont subi certains de leurs collègues. À ce titre, ils apparaissent comme des rescapés d'effets de sélection dus à des conjonctures économiques défavorables (dont celles de marchés professionnels qui les exposent à une concurrence de « gros » ou de « petits ») ou des événements biographiques (divorce, décès, maladie ou accident d'un associé).

2) Ils gagnent ou ont gagné très bien leur vie (« on s'en est bien sorti », selon une expression plusieurs fois répétée). Améliorer leur train de vie a été un objectif premier de leur établissement et sa réalisation se mesure à l'acquisition de biens immobiliers, en plus de leur résidence principale dont ils sont tous propriétaires.

3) Ils sont de très longue date enracinés localement.

Les cinq cas d'enquêtés apparaissant, par contraste, comme minoritaires au regard de ce profil (leur parcours montre une plus grande vulnérabilité aux aléas économiques et biographiques évoqués) ont eu un rôle de confirmation. Ils font exception sur deux points traités en seconde partie de l'article. D'une part, ils n'ont pas pu ou su investir dans la pierre, et le regrettent comme le défaut d'un attribut qui signe la réussite dans le métier à l'heure de la retraite. D'autre part, concernant « le secret de polichinelle » du travail non déclaré, ils entrent aux yeux des «établis » dans la catégorie de ceux qui en abusent.

1. Si le corpus ne compte que deux artisans nés au Portugal, le fait qu'ils fassent référence à d'autres artisans du coin, souvent parents, confirme que le secteur du bâtiment a représenté une perspective locale d'auto-emploi pour une partie de la communauté. 
Une campagne d'entretiens auprès d'experts-comptables s'est imposée dans la mesure où les entretiens avec les artisans les présentaient comme des interlocuteurs incontournables. Des comptables (trois) et experts-comptables (trois) de cabinets implantés autour de la zone d'exercice des artisans enquêtés ont été sollicités en tant qu'acteurs prescripteurs de conseils auprès des artisans. Les comptables rencontrés sont les salariés des experts-comptables. Les premiers sont les interlocuteurs de première ligne pour la tenue régulière des comptes et les incessantes interrogations relatives à des obligations de mise en conformité ; les seconds interviennent à l'occasion de moments et de décisions stratégiques. Le questionnement a veillé à situer les discours tenus par ces acteurs, sans compromettre leur obligation de secret professionnel : sondage du sentiment d'être « représentatif » des experts-comptables sur telle prescription ; indicateurs du poids des artisans du bâtiment dans le portefeuille de clients ; éventail des honoraires.

Dans cette perspective, l'analyse se centre ici sur la figure de l'expert-comptable et sur la prestation intellectuelle que cette profession assure auprès des entreprises artisanales. En complément de l'approche statistique déjà évoquée qui suggère que pour se constituer une retraite, les indépendants persistent à compter sur eux-mêmes et sur le patrimoine qu'ils se constituent (on peut alors parler de conception professionnelle de la retraite), il s'agit de contribuer à une compréhension contextualisée des mécanismes conduisant à une telle persistance. Pourquoi et comment une conception professionnelle de la retraite amène-t-elle à faire passer au second plan, encore aujourd'hui, la pension issue du système par répartition, alors même que l'identité des règles de cotisation et de prestation garantit désormais, et ce depuis 1973 pour les artisans étudiés ici, le même taux de remplacement que dans le régime général pour la retraite de base (encadré 2) ?

L'étude de la place et du rôle de l'expert-comptable permet de repérer deux dimensions distinctes d'une conception professionnelle de la retraite. Une première partie s'attache à la présenter d'abord comme le sous-produit d'une socialisation au statut d'indépendant. Cliché rebattu certes, mais vérité première pour les intéressés, la complexité socio-fiscale les motive majoritairement à recourir à un décrypteur de confiance. S'orienter dans le paysage de possibilités qu'elle dessine commence par le choix éclairé d'une forme juridique d'exercice (voir tableau infra). C'est dans le cadre de ce choix, de sa révision éventuelle au cours du temps, que sont requalifiées du point de vue comptable les cotisations sociales obligatoires, non sans incidence possible sur le montant des cotisations vieillesse. Aussi décisive que soit l'autorité des experts-comptables aux yeux des indépendants enquêtés, elle n'en est pas pour autant absolue. Comme le développe la seconde partie, l'attention sélective que l'indépendant accorde aux conseils de l'expert-comptable perçus comme excédant ceux lui assurant d'être en conformité avec la loi révèle une autre logique professionnelle de mise au second plan de la pension, cette fois-ci liée à la spécificité du métier (mobilisation de ses compétences professionnelles dans le bâtiment pour investir dans la pierre) et non plus au seul statut d'indépendant. 


\section{ENCADRÉ 2}

\section{La retraite en répartition des non-salariés et son alignement sur le régime général}

La notion de taux de remplacement, qui de manière générale rapporte un montant de pension à un montant de revenu d'activité, a des acceptions différentes selon que l'on se place au niveau individuel ou au niveau collectif. Son emploi désigne ici deux aspects importants pour la compréhension du propos.

D'une part, les indépendants n'ont plus de marge de manœuvre pour choisir leur niveau de cotisations sociales au sens où tout leur revenu professionnel déclaré est assujetti obligatoirement aux automatismes de calcul des montants de cotisation en vigueur dans le régime général. L'égalité des taux de cotisation entre salarié et non-salarié signifie que le second doit verser sur son revenu professionnel déclaré un montant représentant la somme de la part employeur et de la part salarié de la Sécurité sociale. Par exemple, au titre de l'assurance vieillesse (de base), en 2014, un salaire brut était assujetti à cotisation sociale, à hauteur de $6,80 \%$ pour la part salariale et de $8,45 \%$ pour la part employeur. En vertu de l'alignement, le non-salarié cotisait donc à cette date à hauteur d'environ $16 \%$ pour sa retraite de base.

D'autre part, second aspect contenu dans l'idée d'égalité de taux de remplacement, un euro cotisé au titre de la retraite de base rapporte un même montant de pension au RSI (non-salariés) et au régime général ( $\mathrm{RG}$; salariés).

La retraite complémentaire obligatoire est la seule composante à faire encore exception dans ce paysage d'égalisation des barèmes de cotisations. La note 3 a déjà indiqué que si le RSI verse aux artisans et commerçants des prestations du même type que les régimes complémentaires des salariés du privé (le montant de la pension complémentaire est le produit d'un nombre de points acquis et de leur valeur au moment de la liquidation), on ne peut cependant pas parler d'alignement dans ce cas car la gestion du régime de retraite complémentaire de ces indépendants continue à obéir à un pilotage financier spécifique. Pour les assurés, cela se traduit par un taux de cotisation analogue à celui appliqué pour la retraite complémentaire des salariés jusqu'à un plafond de revenu (celui de la Sécurité sociale), mais très inférieur au-delà. De $7 \%$, il passe à $8 \%$ au-delà de ce plafond et jusqu'à quatre fois ce plafond, là où pour un cadre, il dépasse $20 \%$ au-delà de ce plafond et jusqu'à huit fois ce plafond.

La retraite complémentaire ne se prête donc pas au même type de raisonnement comparatif que la retraite de base, quant à l'observation des effets sur les comportements des non-salariés de l'application d'automatismes de cotisation venus des régimes des salariés. Le niveau inférieur de prélèvement (au-delà du plafond) explique a priori mécaniquement une partie de la faiblesse persistante relative des pensions des artisans et commerçants, comme l'a rappelé un récent rapport du Haut conseil du financement de la protection sociale (HCFI, 2016, p. 338). Mais force est de reconnaître l'absence de travaux dépassant ce raisonnement mécaniste situé au niveau du régime dans son ensemble.

Concernant les effets sur les comportements, et en particulier ceux de déclaration de ses revenus professionnels, seul niveau où demeure une marge de manœuvre de l'indépendant pour maîtriser le montant de ses cotisations, l'enquête nous a uniquement permis d'identifier une logique comptable, que nous exposons, conduisant à négliger assez radicalement la pension complémentaire sur laquelle l'indépendant pourrait compter. 
Pour les artisans interrogés, l'auto-constitution de leur retraite revêt une importance particulière. Décrivant ce que sont des bonnes conditions de départ à la retraite, ils ont fait valoir sur un ton anodin, parfois amusé, voire ironique, que leur retraite, en fait, ils « se la faisaient». Cette expression, comme celle de «ne pas compter sur la pension » qui lui est régulièrement associée, ne se borne pas à l'ordre du discours. Elle se traduit dans les faits par le montant des revenus locatifs tirés de patrimoines immobiliers que les enquêtés ont bien voulu détailler. De fait, l'idée d'un « complément » apporté à la pension en répartition, qui serait un ajout subsidiaire, apparaît inadéquate pour tous les enquêtés « établis » : alors que leur pension effective ou estimée oscille entre $1500 €$ et $2000 €$, les revenus tirés de leur seul patrimoine immobilier dépassent très souvent cette pension.

«-Votre départ à la retraite, vous l'envisagez comment? Quand?

- Ah mais je n'en sais rien moi!

- Si vous avez 55 ans, vous avez dû recevoir un relevé de carrière avec une estimation de la pension que vous allez toucher à la retraite ?

- Oui.

- Et diriez-vous que vous êtes satisfait?

- Je ne sais pas. Ils m'ont donné le montant brut, je ne sais pas ce qu'il y a comme retenues sur les retraites, alors... Je ne sais même pas comment ça se passe. Je ne sais pas ce qu'ils retirent, je ne sais pas si c'est comme les salaires ${ }^{6}$, je ne sais pas comment ça se passe.

- Mais globalement, vous êtes satisfait ou pas ?

- Oui, euh... Mais moi, de toute façon... je ne comptais pas là-dessus pour vivre hein!

- Vous comptez sur quoi?

- On a un gîte, on a quelques locations quoi. [Rires] Oui, on s'y est préparé un petit peu. »

(Jérôme P., 55 ans, menuisier en entreprise individuelle, trois salariés)

C'est d'abord le fait que nos enquêtés ne semblent pas avoir de connaissances précises sur ce revenu de remplacement qu'est la pension, comme le montre l'extrait ci-dessus, qui nous a conduites à chercher à mieux comprendre un fait connu : la propension des indépendants à assimiler impôts directs et cotisations sociales (ce qu'exprime l'expression contractée : «le socio-fiscal »). Ces deux composantes des prélèvements obligatoires ont en effet en commun pour les indépendants de ne pas être prélevées à la source (BRUNO, 2014, p. 35).

6. C'est nous qui mettons en gras. 


\section{Affronter le socio-fiscal, sa complexité et son poids}

Devenir indépendant, c'est faire l'expérience de l'impossibilité permanente de savoir précisément ce qu'est son revenu professionnel du moment. Choisir de le rester, c'est faire, de plus, celle du changement permanent des règles à respecter pour en connaître le montant officiel.

«Le tiroir-caisse, il n'est pas à nous. Il faut payer les charges, il faut payer tout ce qu'il y a à payer. L'argent, il n'est pas à nous. C'est pour ça que je vous dis qu'il ne faut pas tout mélanger, le tiroir-caisse n'est pas à nous. »

(Chantal M., 62 ans, gérante d'une EI de plâtrerie-peinture, cinq salariés)

« On remue de l'argent, mais cet argent, il n'est pas à nous. Il y en a une partie à l'État, aux impôts, une autre partie en charges maladie et tout ça, donc euh... il y a $1^{\prime}$ Urssaf $^{8}$ si on a des salariés, les fournisseurs... Il y a plein de trucs ! Et puis les lois changent tellement vite... Donc après il en reste moins hein !»

(Leandro F., 59 ans, menuisier retraité anciennement en SARL, trois salariés)

Régulièrement rapportée en entretien, cette double expérience est le fond sur lequel la relation de l'artisan à son expert-comptable peut être décrite comme la socialisation d'un indépendant par un autre indépendant.

\section{L'équivalent du « médecin de famille » pour la petite entreprise}

Profession socialement discrète, quasi ignorée par la sociologie (RAMIREZ, 2005), le comptable libéral, dans la version de praticien local rencontrée lors de l'enquête, est, pour les artisans, un interlocuteur aussi incontournable pour gérer la « santé » de leurs entreprises que peut l'être un médecin généraliste pour la santé personnelle de ses patients.

La première analogie forte réside dans le moment de vérité que représente le bilan comptable qui peut être rapproché d'un bilan de santé. C'est à cette occasion annuelle que le résultat de l'entreprise et les revenus professionnels de l'indépendant, méconnus jusque-là, sont arrêtés. Obligation légale de déclaration, le bilan obéit à des normes impératives de présentation des comptes afin de faciliter la tâche des administrations de l'État susceptibles de les contrôler. S'il faut insister sur ce moment de vérité, c'est d'abord parce que la tenue et l'établissement des comptes restent

7. Les entreprises individuelles (EI) regroupent 51,4\% des artisans. En EI, les biens personnels de l'artisan et ceux de l'entreprise ne font qu'un. D'autres choix sont possibles, puisque 31,8\% exercent en société à responsabilité limitée (SARL), qui permet de dissocier son patrimoine personnel de son patrimoine professionnel : l'artisan détient des «parts » de sa société. L'entreprise unipersonnelle à responsabilité limitée (EURL) (11,9\%) est une variante de la SARL pour un gérant seul détenant l'intégralité des parts de sa société (source : Direction générale des entreprises (2016), Chiffres clés de l'artisanat, édition 2016, Paris, Ministère de l'Économie, de l'Industrie et du Numérique). Les enjeux du choix du statut juridique sont traités plus loin (voir commentaire du tableau, infra).

8. Union de recouvrement des cotisations de la Sécurité sociale et d'allocations familiales. 
la prestation principale pour laquelle les indépendants « classiques $^{9} »$ recourent de manière volontaire à un expert-comptable ${ }^{10}$ et ensuite, parce qu'en déterminant les revenus professionnels, le bilan fixe dans le même temps l'assiette des cotisations sociales, dont les cotisations vieillesse ${ }^{11}$.

La présentation comptable en bonne et due forme des revenus des artisans est relativement récente. Elle s'est imposée à partir des années 1970, suite au renforcement des obligations comptables des petites entreprises, à l'incitation publique à tenir une comptabilité professionnelle, avec la création de centres de gestion agréés, et à la disparition progressive d'une pratique aussi ancienne que prégnante de détermination « forfaitaire » des revenus soumis à prélèvements obligatoires ${ }^{12}$ (BovignY, 1995). Profession réglementée, l'expertise-comptable bénéficie depuis 1945 d'un monopole d'exercice sur la tenue et l'établissement des comptes des entreprises, monopole légal qui s'est cependant accommodé d'un modus vivendi avec les centres de gestion précités, autorisés de fait à proposer la même prestation ${ }^{13}$. Ces prestataires locaux ont tous, centres agréés comme cabinets comptables, développé une offre de services plus globale (Dietrich, Moysan-LouAzel, 2012). Les prestations de conseil juridique, fiscal et social aux entreprises sont reconnues au titre de missions accessoires des experts-comptables, et sont de ce fait soumises aux règles édictées par l'Ordre des experts-comptables.

Comme le soulignent Ève ChiAPELlo et Carlos RAmiRez (2004, p. 3) à propos du désintérêt général de la sociologie pour la comptabilité :

« Paradoxalement, la faculté qu'a la comptabilité de produire une représentation de la "réalité économique" n'intéresse que lorsque cette représentation est entachée de l'enrichissement de dirigeants douteux sur le dos d'actionnaires crédules ou de bailleurs de fonds trompés avec la complicité des auditeurs. On ne s'aperçoit pas que les chiffres des bilans et des comptes de résultat contribuent aussi, en temps normal, à construire cette réalité. »

Cette expertise comptable «normale » s'impose aux indépendants avec l'autorité que peut avoir, pour filer la métaphore, un diagnostic médical basé sur une analyse de sang ou un scanner. Là où l'artisan peine à comprendre le langage technique des

9. L'expression « indépendants classiques » s'est imposée, y compris dans les publications institutionnelles de l'Insee et du RSI, par opposition à celle d'auto-entrepreneurs. L'auto-entrepreneuriat est un régime dérogatoire du statut d'EI créé en 2009 et fondu depuis 2016 dans celui de la microentreprise. Conditionné au non-dépassement d'un seuil de chiffre d'affaires, il dispense l'indépendant de l'établissement d'un bilan comptable, et l'autorise à s'acquitter de son impôt et de ses cotisations sociales par calcul d'un pourcentage forfaitaire de son chiffre d'affaires déclaré.

10. Contrairement au commissaire aux comptes (auquel les grandes sociétés doivent réglementairement faire appel pour contrôler la « sincérité » de leurs comptes), l'expert-comptable, dans le cadre de sa prestation commerciale de tenue des comptes, n'en certifie pas l'authenticité, mais atteste «leur vraisemblance et leur cohérence ».

11. Entre mars et juin, dans le cadre de la déclaration sociale des indépendants (DSI), l'expert-comptable doit communiquer au RSI les revenus de l'année précédente déterminés par la clôture du dernier bilan comptable de son client. 12. Ce dispositif fiscal, extrêmement durable (1934-1999), et ses avatars contemporains sous la forme de l'autoentreprenariat/microentreprise, sont évoqués en seconde partie.

13. L'adhésion à un centre de gestion agréé pour la tenue des comptes ne dispense toutefois pas de les faire attester par un expert-comptable, professionnel avec lequel les centres de gestion ont donc pour obligation de coopérer. 
opérations comptables, le professionnel du chiffre, lui, voit la réalité des fondamentaux faisant la rentabilité d'une activité.

« À force de faire la même chose, on la connaît sur le bout des doigts. Forcément, derrière, il y a un travail de traduction. Je savais leur en parler comme, je ne sais pas, s'ils lisaient un journal. Nous, on voit toute de suite si le client est rentable ou pas. Pour faire simple, s'il n'y a pas de bénéfices, ça veut dire que soit les achats de matières premières sont trop nombreux, soit que les frais généraux sont exagérés, soit qu'on manque de chiffre d'affaires. Ensuite, je leur demandais combien d'heures ils travaillaient et je leur faisais une reconstitution de leur chiffre d'affaires : tant d'heures par semaine par tant de semaines par, allez, $40 €$ HT [hors taxe] de l'heure, ça faisait tel chiffre d'affaires ; les achats consommés ça faisait tant, un coefficient : "Qu'est-ce que vous prenez comme marge ?", "30\%." Hop, ça donne un chiffre d'affaires de marchandises vendues. [...] Voilà comment on diagnostiquait. »

(Denis J., 62 ans, ancien comptable spécialisé dans la clientèle des artisans du bâtiment)

La particularité de ce réalisme est de s'imposer dans le cadre d'une relation de confiance entre deux indépendants qui est notamment motivée par leur complémentarité fonctionnelle. Omniprésente dans la communication de la profession comptable (il faut « vous décharger de ces contraintes légales pour vous concentrer pleinement sur votre activité »), cette complémentarité s'entend également du côté des indépendants dans leur reconnaissance répétée que « la comptabilité, c'est un métier ». Parce que leur prestation de tenue des comptes implique des échanges sur le long terme avec leurs clients artisans, les experts-comptables la revendiquent comme la relation client la plus légitime pour exercer une pratique désintéressée du conseil. Ce d'autant plus que les concurrents sur le marché du conseil aux entreprises (avocats, conseillers juridiques, fiscalistes, courtiers) apparaissent multiples à leurs yeux. Décrite plus loin, l'optimisation socio-fiscale entre dans cette logique générale du « devoir de conseil » sur laquelle l'expert-comptable engage sa responsabilité ${ }^{14}$ et peut être amené à rendre des comptes ${ }^{15}$. Il s'agit pour lui d'éclairer, à l'intérieur d'un champ des possibles qu'il maîtrise et en tenant compte d'un ensemble de paramètres, les décisions de son client au mieux de ses intérêts sur le long terme. Généraliste de la situation financière et comptable de ses clients, il joue ainsi un rôle d'aiguillage des indépendants face à des « spécialistes » en tous genres, notamment quand ces derniers portent une offre commerciale de produits d'épargne :

14. D'après une disposition du Code de déontologie de la profession désormais transcrite dans l'article 155 du décret du 30 mars 2012 relatif à l'exercice de l'activité d'expertise-comptable.

15. Alors que les divers conseillers et experts démarchant les petites entreprises ne se livrent qu'à des prestations ponctuelles, les experts-comptables insistent sur une pratique du conseil inscrite dans la durée et donc sur laquelle ils peuvent avoir à rendre des comptes à l'avenir ( « S'il y a un contrôle fiscal trois ans après, le prestataire qui avait vendu son conseil à l'entreprise, il aura disparu. Pas son expert-comptable », Cyril D., expert-comptable directeur de cabinet, quatre collaborateurs). 
«Vous avez parfois des assureurs qui veulent vendre des Perco ${ }^{16}$ à outrance, en disant : "C'est bien pour vos salariés, mettez ça." Une cliente qui me dit : "Mon assureur est passé, il m'a dit qu'il fallait que je signe”, je lui réponds : "Certes, sauf que des salariés, vous en avez tant, et dans votre cas, ce n'est pas avantageux. Peut-être que l'assureur a besoin de faire son chiffre, mais ce n'est pas bien pour vous. Par contre, demandez-lui, il a peut-être tel type de contrat qui serait bien mieux pour vous, parce que quand je regarde votre résultat sur les cinq dernières années, vous avez plutôt ça, ça et ça, voilà.” »

(Cyril D., expert-comptable directeur de cabinet, quatre collaborateurs)

L'art du conseil défendu par les experts-comptables nécessite donc d'établir une relation s'inscrivant dans la durée. De fait, il est assez difficile de distinguer cet art du conseil de la prestation principale de tenue des comptes : « le conseil est ce qui contribue à entretenir (comme les petits cadeaux entretiennent l'amitié) la relation principale » (RAMIREZ, 2005, p. 379). Il se nourrit des conversations permettant à l'expert-comptable de mieux connaître son client et son environnement de vie, pour ensuite affiner ses conseils, voire tout simplement en formuler.

« Le conseil adapté, on arrive à le donner quand on connaît le client. [...] C'est parce qu'on les connaît, et qu'on les connaît un peu plus que l'activité professionnelle, qu' on va avoir le conseil adapté : “Oh là attendez, cette année votre fille va faire des études supérieures, il faut qu'on se méfie de votre revenu. Vous avez un revenu qui est correct, mais sans être exceptionnel. S'il augmente, c'est clair que vous n'aurez pas de bourse pour les études. S'il baisse un peu, vous pourrez peut-être en avoir." Effectivement on se dit : "Ça ne sert à rien finalement que je fasse un chantier de plus pour arriver à $1000 €$ de plus de bénéfices dans l'année si je n'ai plus aucune bourse pour payer les études de ma fille qui vont me coûter $6000 €$ cette année et que je n'arriverai pas à payer de ma poche." Les clients nous attendent sur plein de choses, mais à condition qu'on les connaisse et ça, ils ne s'en rendent compte que dans la durée. »

(Cyril D., expert-comptable directeur de cabinet, quatre collaborateurs)

La confiance entre artisans et experts-comptables est également motivée par le rapport d'estime mutuelle entre deux professionnels « bosseurs ». Une quasi-confraternité peut naître du partage de la condition d'indépendant et d'une conception commune du « fruit de leur travail » : « Il faut prendre tout ça en compte. Et puis faire comme si c'était sa boîte quoi, ou la boîte de son fils ${ }^{17}$. » Les experts-comptables se disent d'ailleurs constamment interpellés par cette question de leurs clients : « Et que feriez-vous à ma place ? » Ces formes d'identification - ou d'effet miroir - tiennent en partie à une circularité de la réussite : des experts-comptables expriment leur admiration des artisans du bâtiment, du moins des « bons », autrement dit de ceux qu'ils

16. Les plans d'épargne retraite collective permettent aux chefs d'entreprise employeurs d'abonder un compte d'épargne pour leurs salariés et pour eux-mêmes, en vue de la retraite. L'argent placé est en partie défiscalisable et n'entre pas dans l'assiette des cotisations sociales obligatoires.

17. Alain V., expert-comptable directeur de cabinet, huit collaborateurs. 
considèrent comme « sachant travailler » et « sachant gérer ${ }^{18}$ », les confortant dans le bien-fondé de leur accompagnement.

«On apporte un avis sur tout ce qui est la problématique du chef d'entreprise, tout ce qui paraît difficile pour lui, c'est-à-dire ce qui n'est pas son cœur de métier. Dans son cœur de métier généralement, il excelle et nous, on n'a que des notions. Tout le reste, nous, normalement, on doit exceller, et lui il n'a que des notions. [...] Donc si vous avez un expert-comptable qui est présent et puis un artisan qui l'écoute, c'est vraiment rare qu'il se casse la binette. »

(Alain V., expert-comptable directeur de cabinet, huit collaborateurs)

À ce registre d'intérêt à la réussite de l'artisan ${ }^{19}$, peut s'ajouter le sentiment de participer à une action de justice sociale et fiscale : les experts-comptables arment les « petits », défavorisés par rapport aux « gros » (c'est-à-dire les grandes entreprises qui ont leurs propres services juridiques et recourent aux prestations de cabinets internationaux d'audit) $^{20}$. Aussi les experts-comptables n'hésitent-ils pas à se présenter comme la profession gardienne des intérêts des «petits » : « nous sommes les seuls en fait, nous sommes les seuls professionnels à être les conseils récurrents des entrepreneurs en TPE et en petite $\mathrm{PME}^{21} »$. Ils dénoncent l'illisibilité des dispositifs réglementaires actuels, producteurs selon eux d'inégalités sociales.

«Quand on voit le mal qu'on a à expliquer certaines choses, que des fois il faut réexpliquer, c'est tellement compliqué en fait que c'est inéquitable. Une fiscalité juste, c'est une fiscalité simple. Alors, ce n'est pas que la fiscalité, c'est le social, c'est le juridique... [...] Donc, s'il faut avoir bac +4 pour payer le juste impôt sur le revenu, ça ne devient plus juste, ça ne devient plus équitable. »

(Alain V., expert-comptable directeur de cabinet, huit collaborateurs)

Enfin, eux-mêmes affiliés au RSI (pour la maladie) et à une caisse toujours spécifique aux professions libérales pour la retraite, les experts-comptables interrogés exerçant en SARL partagent largement la condition socio-fiscale de leurs clients. Elle peut les amener à tenir des propos virulents dénonçant la taxation du « fruit de leur travail », jugée inique au regard de la taxation des revenus du grand capital.

\footnotetext{
18. «Les gens qui gagnent de l'argent, c'est des gens qui sont hyper rigoureux et vachement bien organisés, qui passent aussi du temps au boulot hein ! » (Cyril D., expert-comptable directeur de cabinet, quatre collaborateurs).

19. Les honoraires des experts-comptables sont libres ; les pratiques de tarification variables, au forfait annuel révisable ou au temps passé. Pour les missions définies comme de nature comptable, la tarification en fonction du résultat financier du client est interdite. Selon le montant du forfait contractualisé, l'entreprise cliente ne peut s'attendre ni au même niveau de décharge des tâches comptables (degré de délégation du travail en amont d'établissement ordonné des pièces comptables), ni, surtout au-delà, à la même qualité générale de suivi (conseil « gratuit »). Ainsi, dans un cabinet enquêté, pour un artisan exerçant sans salarié, le tarif annuel varie de $1000 €$ à $5000 €$ hors taxe. À $1000 €$, la sollicitation ponctuelle de conseils fera l'objet d'une facturation supplémentaire.

20. Sur les rapports d'homologie entre les différents segments du champ de la profession comptable et leurs clients respectifs, voir RAMIREZ (2005).

21. Très petite entreprise ; petite et moyenne entreprise. Propos d'Alain V., expert-comptable directeur de cabinet, huit collaborateurs.
} 
«Aujourd'hui, on taxe aussi les dividendes en cotisations sociales. Les dividendes, c'est le fruit du capital. Philosophiquement, ça me fait mal au cœur. Parce qu'on considère que pour un gérant majoritaire de SARL, le fruit du capital n'est que le fruit du travail. C'est complètement un déni de justice. Dans les sociétés cotées en Bourse, il est logique de rémunérer un actionnaire. Pas en SARL ! Cherchez l'erreur ${ }^{22}$ ! »

(Alice G., experte-comptable directrice de cabinet, quinze collaborateurs)

Le raisonnement ici rapporté fait référence aux « montages » juridiques déterminant les assiettes sociales et fiscales de dirigeants inégalement grands dans l'ordre économique.

\section{La requalification des cotisations sociales obligatoires dans le cadre de « montages »}

Certains travaux de sociologie portant sur les contribuables ont introduit la notion de montage pour désigner l'existence de dispositifs d'évitement/allégement de l'impôt.

«Du côté du contribuable, la notion de montage est essentielle : la compliance (le conformisme fiscal) ne traduit pas forcément une acceptation morale de l'impôt mais l'absence de décision effective d'éviter l'impôt. L'analyse doit partir de l'évitement de l'impôt plutôt que de postuler le civisme. »

(LEROY, 2003, p. 218)

Une telle perspective invite à s'intéresser aux occasions et aux conditions pratiques dans lesquelles se joue un consentement à s'acquitter des prélèvements obligatoires. Elle est d'autant plus judicieuse que dans le cas du rapport au socio-fiscal des artisans qui nous intéresse ici, les opportunités de réduire le montant de leurs prélèvements obligatoires (les montages) sont indissociables de choix impératifs liés au statut d'indépendant.

Le premier choix à effectuer est bien évidemment celui du statut d'exercice au moment de la création de l'entreprise ${ }^{23}$ (tableau 1). Pour les indépendants « classiques », un tel choix implique en même temps celui du régime fiscal et du régime social.

22. Les propos tenus par Alice G. soulignent la double nature des revenus professionnels des indépendants (revenus à la fois du travail et du capital). Cette nature mixte soulève des difficultés pour leur mode d'assujettissement aux prélèvements obligatoires. Les dividendes sont traités différemment selon le statut social des travailleurs indépendants. Pour les « assimilés salariés » (catégorie dont relèvent notamment les présidents directeurs généraux des sociétés cotées en Bourse mentionnés par l'enquêtée), ils sont considérés comme des revenus de capitaux mobiliers et ne sont donc pas soumis à cotisations sociales, tandis que pour les gérants majoritaires de SARL affiliés au RSI (cas de l'enquêtée), les dividendes sont assujettis à cotisations sociales (pour la fraction supérieure à $10 \%$ du capital social). Cette différence de traitement entre catégories de travailleurs indépendants a d'ailleurs été soulignée par un récent rapport du Haut Conseil du financement de la protection sociale proposant d'étendre la mesure d'assujettissement des dividendes aux dirigeants assimilés salariés, « en regard de l'unicité de traitement qui prévaut (par ailleurs) en matière fiscale » (HCFI, 2016, p. 330).

23. Cf. note 7 sur la répartition des artisans par statut d'exercice. 
TABLEAU 1 - Régime fiscal et régime social des indépendants selon leur statut juridique d'exercice

\begin{tabular}{|c|c|c|c|c|}
\hline Statut juridique & Régime fiscal & Assiette fiscale & Régime social & Assiette sociale \\
\hline $\begin{array}{l}\text { Entrepreneur } \\
\text { individuel (hors } \\
\text { microentrepreneur) }\end{array}$ & $\begin{array}{l}\text { Catégorie } \\
\text { «Bénéfices } \\
\text { industriels et } \\
\text { commerciaux » } \\
\text { (BIC) } \\
\text { Non-salarié }\end{array}$ & $\begin{array}{l}\text { Bénéfice (net de } \\
\text { cotisations) soumis } \\
\text { à l'impôt sur le } \\
\text { revenu }\end{array}$ & $\begin{array}{l}\text { Régime social des } \\
\text { indépendants (RSI) } \\
\text { Non-salarié }\end{array}$ & Bénéfice \\
\hline $\begin{array}{l}\text { Présidents, } \\
\text { directeurs généraux } \\
\text { de SA, de SAS }{ }^{*} \text { et de } \\
\text { SASU }^{* * *}\end{array}$ & $\begin{array}{l}\text { Catégorie } \\
\text { «Traitements et } \\
\text { salaires » } \\
\text { Assimilé salarié }\end{array}$ & $\begin{array}{l}\text { Rémunération du } \\
\text { gérant soumise } \\
\text { à l'impôt sur le } \\
\text { revenu. } \\
\text { Reste du bénéfice } \\
\text { dans la société } \\
\text { soumis à l'impôt } \\
\text { sur les sociétés }\end{array}$ & $\begin{array}{l}\text { Régime général } \\
\text { (RG) } \\
\text { Assimilé salarié }\end{array}$ & $\begin{array}{l}\text { Rémunération } \\
\text { du gérant }\end{array}$ \\
\hline $\begin{array}{l}\text { Gérant majoritaire } \\
\text { de SARL et gérant } \\
\text { unique d'EURL }\end{array}$ & $\begin{array}{l}\text { Catégorie } \\
\text { "Rémunérations } \\
\text { des dirigeants } \\
\text { assimilées à un } \\
\text { salaire » } \\
\text { Assimilé salarié }\end{array}$ & $\begin{array}{l}\text { Rémunération du } \\
\text { gérant soumise } \\
\text { à l'impôt sur le } \\
\text { revenu. } \\
\text { Reste du bénéfice } \\
\text { dans la société } \\
\text { soumis à l'impôt } \\
\text { sur les sociétés }\end{array}$ & $\begin{array}{l}\text { Régime social des } \\
\text { indépendants (RSI) } \\
\text { Non-salarié }\end{array}$ & $\begin{array}{l}\text { Rémunération } \\
\text { du gérant et } \\
\text { dividendes (sur la } \\
\text { fraction supérieure } \\
\text { à } 10 \% \text { du capital } \\
\text { social détenu) }\end{array}$ \\
\hline
\end{tabular}

* Société par actions simplifiée.

** Société par actions simplifiée unipersonnelle.

Comme l'indique le tableau, la pluralité des configurations socio-fiscales des indépendants a pour corollaire celle des assiettes. Ainsi, un entrepreneur individuel paye ses cotisations sociales sur la base de ses bénéfices, tandis que celles d'un gérant d'une $\mathrm{SAS}^{24}$ sont calculées sur la base de sa rémunération. Concernant les gérants majoritaires de SARL, une partie des dividendes est intégrée à l'assiette sociale depuis 2013 (voir supra, note 22). Dans cet éventail de statuts d'exercice, on note la possibilité dans les cas de formes sociétaires hors SARL (deuxième ligne du tableau) d'être un « assimilé salarié », catégorie dont l'indépendant peut souhaiter relever afin d'être couvert par le régime général (RG), en particulier dans un contexte de défiance vis-à-vis du RSI ${ }^{25}$. Les experts-comptables interrogés ne

24. La société par actions simplifiées (SAS) et sa variante unipersonnelle (SASU) ont enregistré un succès récent : en 2016, plus de la moitié des sociétés créées sont des SAS (BONNETÊTE, BiGNON, 2017).

25. Menés par le mouvement «Sauvons nos entreprises » créé en 2011, des collectifs de travailleurs indépendants se sont en effet constitués ces dernières années pour contester les dysfonctionnements du RSI, et notamment les procédures de recouvrement d'impayés de cotisations sociales à la suite de la mise en place de l'Interlocuteur social unique en 2008, la gestion du recouvrement ayant ainsi été confiée aux Urssaf pour le compte du RSI. D'autres vont même jusqu'à rouvrir un dossier législatif ancien, à l'initiative du Mouvement des libérés (de la Sécu), contestant l'existence légale du RSI, et plus généralement des régimes de protection sociale obligatoire en France (sur ce point précis, voir « RSI : le nouvel argument choc des Libérés pour faire tomber le régime », L'Express, 15 décembre 2016). Les manifestations récentes contre le RSI ont fait l'objet d'une large couverture médiatique par la presse nationale 
souscrivent pas à une telle défiance, dans la mesure où ils déconseillent généralement le statut d'assimilé salarié parce qu'ils le jugent inadapté à la majorité de leurs clients. Sollicités par les petits indépendants sur le choix de leur statut d'exercice, ils les éclairent en produisant des raisonnements chiffrés. C'est dans ce cadre qu'ils font valoir l'affiliation au RSI comme plus économique que l'affiliation au RG (encadré 3).

\section{ENCADRÉ 3}

\section{Plaidoyers d'experts-comptables pour le RSI}

Nous reproduisons ci-dessous un extrait d'entretien avec Cyril D., expert-comptable directeur de cabinet, 35 ans, quatre collaborateurs :

«-Et vous-même, comment vous positionnez-vous sur cette question du choix de la forme juridique d'exercice?

- Moi, je suis un peu à contre-courant de ce que répandent les Chambres de commerce ou autres sur la création. Beaucoup de gens viennent en disant : "Je reviens de la Chambre de commerce, ils m'ont dit de me mettre en SAS parce que comme ça, je peux être salarié de ma structure et que cela ne me coûtera pas beaucoup de charges sociales. On m'a dit que c'était moins cher que le RSI." Je réponds : "OK, on va faire une simulation. Si vous êtes président de votre SAS à $1000 €$ par mois, votre SAS va avoir des charges sociales pour à peu près l'équivalent de votre salaire. Alors que si vous êtes en SARL au RSI, [...] objectivement, si vous gagnez $1000 €$, vous aurez un RSI qui va vous coûter $500 €$, deux fois moins". »

Extrait d'un autre entretien, cette fois avec Alain V., expert-comptable directeur de cabinet, 58 ans, huit collaborateurs :

« - La forme juridique qu'on conseille le plus, c'est la SARL. Pour un problème de coût. Ce qui est très important quand une entreprise démarre, c'est qu'elle fasse des économies. Et ce qui est difficile - pour nous aussi -, c'est que quand on fait une SARL à gérance majoritaire, et donc que les gens sont au RSI, il faut leur expliquer pourquoi le RSI c'est bien, alors que dans le même temps, il y a des gens qui défilent contre le RSI ! Pas facile hein ! Et quand je les vois défiler contre le RSI, je me dis : "Mais ces cons-là, ils vont finir par nous faire tomber le régime qui est quand même le plus favorable !"

- Le plus favorable?

- Bien sûr!

- Alors expliquez-moi...

- C'est très simple. Vous vous donnez une rémunération nette de $10000 €$ dans une SARL. En tant que gérant majoritaire, ou en tant qu' entrepreneur individuel,

(voir, entre autres, les titres suivants : « Le RSI, cauchemar des travailleurs indépendants », Les Échos, 28 avril 2014 ; « Pourquoi les travailleurs indépendants fustigent le RSI », Le Monde, 11 mars 2015 ; « RSI : "Les gens n'en peuvent plus" », L'Express, 28 novembre 2016). 
c'est le même régime, celui du RSI. Donc vous avez une pression sociale obligatoire, y compris la $\mathrm{CSG}^{1}$ qui est considérée comme une taxe, de $50 \%$, c'est-à-dire que ces $10000 €$ nets de rémunération vont engendrer $5000 €$ de cotisations au RSI. Maintenant, on va faire notre président de SAS (affilié au RG), le gars qui, sur les bons conseils de je ne sais pas qui, a voulu démarrer en SAS, et qui se donne sur une période déterminée $10000 €$ de rémunération nette, il paye 8700 ! Pression sociale obligatoire : 8700 . Vous voyez l'écart ? Et encore, il ne paye pas d'Assédic ${ }^{2}$ puisqu'il est au régime général mais en assimilé salarié. [...] »

Dans ces deux occurrences, le raisonnement part du revenu disponible visé par le dirigeant et compare les coûts de protection sociale qu'il génère pour l'indépendantentreprise selon que ce dirigeant est non-salarié ou assimilé salarié.

Explicitons ainsi les chiffres exposés dans le second extrait d'entretien.

Dans le premier cas, celui de l'indépendant, en EI ou SARL, les cotisations sociales s'élèvent à $5000 €$ (correspondant à l'ordre de grandeur d'un taux de $50 \%$, confirmé dans les publications du $\mathrm{RSI}^{3}$ ).

Dans le second cas, celui du dirigeant salarié d'une société, le montant avancé de $8700 €$ correspond à la somme de la part salariale et de la part patronale des cotisations. Une rémunération nette de $10000 €$ correspond à une rémunération brute de $12820 €$. La part salariale est de $2820 €$ ( $22 \%$ du brut). La part patronale, versée par la société, donc toujours par lui pour les comptables, correspond à $45 \%$ du brut, soit environ $5800 €$. Le montant arrondi de 8700 résulte de l'addition de ces deux composantes $(2820+5800)$.

1. Contribution sociale généralisée.

2. Association pour l'emploi dans l'industrie et le commerce.

3. Voir notamment le tableau présentant le pourcentage des cotisations RSI sur la base d'un revenu net de $20000 €($ Rsi, 2015, p. 40).

La démonstration d'un coût global de sa protection sociale plaidant pour le choix du RSI se place donc du point de vue du poids financier que ce coût représente pour l'homme-entreprise pris comme un tout indissociable. Une fois le choix du RSI acté, les experts-comptables peuvent se livrer à des calculs relevant de l'optimisation socio-fiscale ${ }^{26}$. Les cotisations sociales obligatoires, dont les cotisations vieillesse, sont alors traitées dans le cadre d'un calcul coût-bénéfice. Ce type de calcul est apparu dans l'enquête comme indissociable, de nouveau, d'un moment de choix de statut d'exercice par l'indépendant : notamment celui où, les bénéfices d'une petite entreprise individuelle augmentant, sa transformation en SARL s'impose d'un point de vue comptable comme permettant un jeu entre assiette sociale et assiettes fiscales (c'est-à-dire la sienne en tant que particulier contribuable et celle de son entreprise au titre de l'impôt sur les sociétés). Un comptable (encadré 4) a expliqué de manière très pédagogique comment, à l'occasion d'un tel changement, il apprécie ce que rapportent

26. Pour rappel, « distincte de la fraude, qui présente un caractère délictuel, l'optimisation consiste à minimiser le prélèvement que l'on doit payer en utilisant les différents dispositifs légaux » (WANECQ et al., 2008, p. 102). 
les différentes cotisations par rapport à ce qu'elles coûtent. Rappelons que, comme dans le régime général, la cotisation des non-salariés au titre de leur retraite obligatoire de base ne donne pas de droits supplémentaires au-delà du plafond de la Sécurité sociale (autour de $38000 €$ annuels en 2015-2016). Autrement dit, seuls les revenus en deçà du plafond sont pris en compte pour le calcul de la retraite de base. C'est l'une des conséquences-traductions de l'alignement de la protection sociale des non-salariés (RSI) sur celle des salariés (RG).

\section{ENCADRÉ 4}

\section{Hiérarchisation des cotisations et référence cible du plafond de la Sécurité sociale}

Nous reproduisons ci-dessous l'extrait d'un entretien avec Denis J., comptable nouvellement retraité de 62 ans.

«- Un gars qui travaille tout seul peut très bien gagner $60000,80000 €$ par an. À ce moment-là, il ne faut plus rester en entreprise individuelle, il faut passer en société. Là, il est gérant, et donc les cotisations RSI, au lieu d'être basées sur les 80 000, elles vont être basées sur la rémunération que le gérant va vouloir se fixer. Par exemple, il se dit : "Ben moi, pour bien vivre, il me faut $3000 €$ par mois." Donc $3 \times 12=36$, voilà ma rémunération. Les cotisations RSI vont être basées sur $36000 €$. Et le reste va être soumis à l'impôt sur les sociétés ${ }^{1}$. Voilà, plutôt que de payer dans le vide.

La pension de retraite de l'artisan est basée, comme pour un salarié, au maximum sur le plafond de la Sécurité sociale. Il est cette année de 38 616, donc voisin des 36000 que j'ai pris dans mon exemple. Donc, le but du jeu, c'est qu'il ait une rémunération en tant que gérant au moins égale au plafond, de façon à ce que sa cotisation de retraite de base soit au taquet. Donc, il faut qu'il ait une rémunération annuelle d'au moins $38616 €$ en 2016. [...]

S'il ne passe pas en société, il va payer beaucoup, beaucoup de cotisations au RSI sans que cela ne lui rapporte rien. Il faut bien voir qu'il y a trois types de cotisations :

- la cotisation allocations familiales, donc ça va à l'Urssaf, elle est redistribuée à tous les gens qui ont des enfants, allocations logements, bon, les prestations sociales familiales, quoi ;

- la cotisation maladie sert de couverture santé, comme pour les salariés, mais là, que tu cotises plus ou moins, tu es remboursé pareil. Donc si tu as $120000 €$ de résultat, tu vas payer des cotisations maladie sur $120000 €$ pour les autres, ça ne te rapporte rien ;

1. La possibilité de se fixer un montant choisi de rémunération en SARL permet, au dirigeant en tant que gérant, de contrôler le montant de son assiette sociale (égale à la rémunération), et donc de ne pas cotiser sur l'ensemble des bénéfices comme c'est le cas en entreprise individuelle où la distinction entre rémunération et bénéfice n'existe pas. De la même manière, elle permet de contrôler l'assiette de l'impôt sur le revenu, pour la part égale à la rémunération, tandis que les bénéfices non prélevés par le dirigeant seront quant à eux soumis à l'impôt sur les sociétés (cf. supra tableau). 
- et puis la cotisation retraite, c'est différent parce que tu payes pour toi, mais ta cotisation retraite elle est plafonnée au plafond de la Sécu, alors même si tu as $120000 €$ de bénéfice, ta cotisation retraite de base, elle va être basée sur 38616 ;

- après il y a la cotisation retraite complémentaire qui existe bien au-delà du plafond du régime de base, jusqu'à $154464 €$ en 2016, c'est-à-dire que là, on a une cotisation de $8 \%$. Alors évidemment, c'est intéressant de ce côté-là, mais il y a beaucoup plus de côtés inintéressants pour la cotisation allocations familiales, la cotisation maladie, que de côtés intéressants. Alors c'est bête de payer des cotisations pour le pot commun qui ne te rapportent rien. Il vaut mieux limiter la cotisation retraite de base, c'est-à-dire au plafond de $38616 €$ et puis utiliser d'autres systèmes [comme la] loi Madelin qui sont déductibles fiscalement ${ }^{2}$, ou d'autres investissements de façon à se créer des retraites perso, plutôt que de payer des cotisations allocations familiales et maladie. C'est utiliser l'argent autrement.

- Donc si je comprends bien, on fait l'impasse sur la retraite complémentaire RSI, et il vaut mieux faire sa retraite autrement?

- Voilà. Ce n'est pas que la cotisation complémentaire RSI n'est pas intéressante, mais elle oblige à cotiser sur d'autres postes qui ne rapportent rien. C'est ça le problème. »

2. Depuis une loi de même nom de 1994, le cadre dit Madelin encourage l'auto-protection sociale par une défiscalisation spécifique aux indépendants. Il les autorise à déduire de leur revenu professionnel imposable l'épargne qu'ils placent volontairement auprès d'organismes privés de prévoyance. Sont concernées non seulement la retraite supplémentaire mais aussi la mutuelle santé, l'assurance perte d'emploi et l'assurance indemnités journalières.

Que retenir du raisonnement comptable rapporté ? Tout d'abord, il est conditionné par un ordre de grandeur : le niveau des bénéfices (entre 60000 et $80000 €$ ) détermine le conseil donné aux travailleurs indépendants de transformer leur entreprise individuelle en SARL. Ensuite, le raisonnement soupèse des taux automatiques de cotisation (pourcentage et plafond fixes par risque) pour faire jouer à l'assiette déclarée le rôle de variable d'ajustement afin de garantir un meilleur rendement des cotisations individuelles. Déclarer un revenu professionnel approchant le plafond de la Sécurité sociale assure ainsi l'acquisition du maximum possible de droits pour la retraite de base, tout en évitant de cotiser au-delà « à perte » pour les postes sans plafond (maladie et famille). Cette hiérarchisation des catégories de cotisations conduit à négliger la pension complémentaire dont les cotisations obligatoires s'appliquent jusqu'à quatre fois le montant du plafond (et déterminent en conséquence une pension totale plus élevée). Il y a donc une incitation - objectivée par les calculs techniques d'optimisation - à renoncer à une partie de la pension en répartition.

Intéressant en tant que tel, ce premier niveau de lecture n'est cependant pas à lui seul suffisant. Il convient en effet d'être attentif aux conditions dans lesquelles le raisonnement comptable est produit. Pédagogique à l'intention d'enquêtrices non 
spécialisées en matière socio-fiscale, le raisonnement l'est à la mesure du nombre de paramètres que les comptables prennent en compte pour s'ajuster à la spécificité de chaque cas d'homme-entreprise. À l'intérieur même d'une mise en balance la plus pure possible de « ce que ça coûte » versus « ce que ça rapporte », les experts-comptables ont besoin de recourir à des logiciels dédiés de simulation ${ }^{27}$. De plus, ils sont tenus par leur déontologie de profession réglementée de produire des revenus déclarés en adéquation avec le «train de vie » des indépendants et leur ménage, reconstituable par l'administration fiscale. Enfin, et peut-être surtout sociologiquement, le conseil de « cotiser au plafond » (de la Sécurité sociale), décrit plus haut, s'éclaire plus largement par la mission d'alerte des experts-comptables sur les éventualités pour leur client de travailler et déclarer plus « pour rien », « à perte », ou selon une expression synonyme pour ces acteurs, « pour l'État».

\section{S'en remettre à son comptable pour mieux s'en détacher}

Nous avons montré que la médiation comptable pouvait opérer comme une première logique de mise au second plan de la pension RSI, s'inscrivant dans un cadre de socialisation au statut d'indépendant. Mais qu'en est-il de la perception, de la réception et de l'évaluation, par les intéressés, du travail de l'expert-comptable et de ses salariés ? Nous proposons de nous intéresser à leurs modalités car elles font intervenir les référents professionnels propres aux artisans du bâtiment interrogés. Leur analyse permet de comprendre comment le sens de la retraite s'enracine aussi dans le métier, dont l'exercice a une capacité propre à faire intérioriser le caractère secondaire de la pension par répartition.

\section{Compliance work de l'expert-comptable et assurance de « cotiser sérieusement » pour l'indépendant}

S'en remettre à son expert-comptable découle d'une certaine façon de la complémentarité fonctionnelle notée plus haut. L'expert-comptable est d'abord et avant tout perçu comme l'agent d'un compliance work, c'est-à-dire d'une mise en conformité avec les obligations fiscales et sociales (RAMIREZ, 2005). Travail essentiel puisque le montant de sa prestation est volontiers assimilé par les intéressés au prix à payer pour leur tranquillité d'esprit. Une composante de base de cette tranquillité se loge dans la conviction qu'avoir un expert-comptable (versus ne pas en avoir) aide à passer en

\footnotetext{
27. L'intérêt de la gérance majoritaire de SARL ne se réduit pas simplement à une minimisation de sa rémunération (au plafond de la Sécurité sociale) qui lui permettrait d'augmenter les dividendes perçus. Il prend en compte l'impôt sur les sociétés (IS) versé par l'entreprise et l'impôt sur le revenu (IR) dont le dirigeant est redevable aussi sur la part des bénéfices qu'il se reverse (dividendes). L'intégration récente (2013) des dividendes dans l'assiette sociale, décriée par l'experte-comptable citée p. 55, a complexifié un peu plus les paramètres de l'optimisation. D'où l'équipement en logiciel idoine.
} 
dessous du radar du fisc. La hantise du contrôle fiscal et la certitude qu'il se solde immanquablement par un redressement sont exprimées de manière récurrente (« [Les agents du fisc] ne viennent jamais pour rien ! Le temps qu'ils passent, faut bien que ça produise une facture $\left.{ }^{28} \gg\right)$. À cela, s'ajoute une préoccupation majeure, exprimée par les artisans du corpus, relative au respect de leurs obligations sociales, et plus précisément au provisionnement de leurs cotisations sociales. Pour bien comprendre les questions qui se posent aux artisans, il importe de préciser le calcul et le règlement des cotisations RSI dans toute leur technicité. Cette technicité détermine non seulement une occasion privilégiée pour les artisans de s'en remettre à leur comptable mais elle leur procure également dans le même temps un sentiment légaliste de « cotiser sérieusement », et ce par rapport à un premier référent professionnel : leurs aînés.

Le coût de sa protection sociale se rappelle à l'indépendant sous la forme d'un échéancier annuel de cotisations transmis par le RSI dans un « avis d'appel » (tableau 2).

TABLEAU 2 - Exemple d'échéancier d'appel de cotisations d'un assuré du RSI «en régime de croisière ${ }^{29}$ "

En euros

\begin{tabular}{lccc}
\hline Période & $\begin{array}{c}\text { A. Cotisations provisionnelles } \\
\mathbf{2 0 1 6} \text { (annexe 2) }\end{array}$ & $\begin{array}{c}\text { B. Régularisation définitive } \\
\text { des cotisations 2015 (annexe 1) }\end{array}$ & $\begin{array}{c}\text { C. Montant restant } \\
\text { à payer en 2016 }\end{array}$ \\
\hline 05 janvier & 2395 & $/$ & $/$ \\
05 février & 2395 & $/$ & $/$ \\
07 mars & 2395 & $/$ & $/$ \\
05 avril & 2395 & $/$ & $/$ \\
06 mai & 2395 & $/$ & $/$ \\
06 juin & 2395 & 441 & 3319 \\
05 juillet & 2878 & 441 & 3319 \\
05 août & 2878 & 441 & 3319 \\
05 septembre & 2878 & 441 & 3319 \\
05 octobre & 2878 & 441 & 3319 \\
05 novembre & 2878 & 433 & 3313 \\
05 décembre & 2880 & $\mathbf{2 6 3 8}$ & $\mathbf{1 9} 908$ \\
TOTAL & $\mathbf{3 1 6 4 0}$ & & $/$ \\
\hline
\end{tabular}

Source : échéancier d'appel de cotisations reçu en juin 2016 par un artisan du bâtiment du corpus âgé d'une cinquantaine d'années, à son compte depuis plus de vingt ans et ayant transformé son entreprise individuelle en société en 2011 (extrait d'un matériau recueilli sur le terrain le 10 août 2016).

Le dispositif d'appel des cotisations RSI repose sur trois mécanismes impliquant un recalcul des cotisations tous les six mois. Au cours du premier semestre d'une année civile N (ici, 2016), un ressortissant du RSI « provisionne » des cotisations sur la base de son revenu $\mathrm{N}-2^{30}$. La connaissance du revenu réel de $\mathrm{N}-1$ en milieu d'année $\mathrm{N}$

28. Alberto C., 59 ans, ancien associé d'une société de maçonnerie devenu marchand de biens, solo.

29. La catégorie « en régime de croisière » du RSI désigne les assurés ayant plus de trois ans d'activité.

30. Pour des raisons de délai d'établissement des déclarations réglementaires, non seulement le revenu de l'année N (2016) ne sera réellement connu qu'au milieu de N + 1 (2017) mais, pour la même raison, le revenu N - 1 (2015) n'est connu qu'au milieu de l'année $\mathrm{N}$. 
produit un double effet. Le premier est rétrospectif. Il correspond au mécanisme ancien de la « régularisation ». Une fois ce revenu réel $\mathrm{N}-1$ connu, le RSI revient en arrière pour reconsidérer la justesse du total des cotisations qui ont été provisionnées en $\mathrm{N}-1$ (sur la base des revenus de $\mathrm{N}-3$ ). C'est ce qui conduit l'indépendant à faire un versement supplémentaire dans l'échéancier donné en exemple (deuxième colonne). L'indépendant solde donc le coût réel de sa protection sociale de l'année passée $\mathrm{N}-1$ (ici, 2015), au cours de la seconde partie de l'année N. Le second effet est prospectif et correspond à un mécanisme récent, celui du « réajustement » en cours d'année $\mathrm{N}$ des cotisations provisionnelles. Il se traduit dans la première colonne de l'échéancier par un changement du montant à partir de juillet. En effet, afin d'éviter une régularisation importante en $\mathrm{N}+1$, en cas de fortes variations de revenus, le RSI réajuste au milieu de l' année $\mathrm{N}$ les cotisations provisionnelles sur la base des revenus $\mathrm{N}-1$ alors connus. L'appel des cotisations est donc un dispositif complexe d'enregistrement. Il repose sur trois mécanismes - le «3-en-1 » du provisionnement, de la régularisation et du réajustement -, mécanismes qui tuilent un calcul de cotisations engageant lui-même en continu les revenus de trois années.

En dépit des réformes du dispositif visant à lisser les écarts dans les montants appelés, ce dernier reste marqué par des versements au RSI aux montants variables, ce qui redouble, en raison de la temporalité décalée de ces versements par rapport aux revenus, l'expérience d'une activité pouvant alterner les « bonnes » et les « mauvaises » années. Les entretiens expriment avec force onomatopées le poids que représente un appel ou une régularisation («Et là, boum boum boum ! », « Bing ! », «Paf ! Grand coup de masse sur la tête !»). Le caractère indépassable de cette variabilité (des montants appelés) tient à la connaissance ex post des revenus servant d'assiette sociale (à la clôture du bilan) et à leur délai de traitement par le $\mathrm{RSI}^{31}$. Face à la révision bi-annuelle du montant de leurs cotisations, les indépendants sont contraints d'assurer un suivi régulier de leur trésorerie afin d'éviter le risque d'un défaut de paiement. On comprend ainsi pourquoi le comptable est, comme pour toutes les dépenses à provisionner avec rigueur, l'interlocuteur auquel l'artisan s'en remet pour la gestion experte de son tiroir-caisse que rend nécessaire l'acquittement de ses cotisations sociales.

Paradoxalement, les contraintes calculatoires imposées aux indépendants par l'appel de cotisations, et pour se décharger desquelles ils rémunèrent les services d'un expert-comptable, ne sont pas rapportées dans des termes « poujadistes » porteurs d'une critique aveugle du RSI qui serait considéré comme le bras armé d'une pression

\footnotetext{
31. Parmi les organisations siégeant au conseil d'administration du RSI, la Confédération générale des petites et moyennes entreprises (CGPME) s'est distinguée en plaidant, depuis 2015, pour une solution présentée comme radicale : l'auto-liquidation des cotisations obligatoires (auto-déclaration et auto-paiement) pour les ressortissants du RSI. L'assuré calculerait et verserait lui-même ses cotisations sur la base de ses revenus actuels, sans médiation d'un avis d'appel adressé par le RSI. Des rapports institutionnels ont examiné cette proposition, soit pour se prononcer en faveur d'une expérimentation de l'auto-liquidation (Bulteau, Verdier, 2015 ; Cese, Weber, 2015), soit pour en écarter toute mise en œuvre (IGAS, IGF, 2016).
} 
sociale aussi arbitraire que démesurée. C'est un résultat important de l'enquête que d'enregistrer un légalisme des indépendants établis que nous avons rencontrés :

«C'est vrai que tous ceux qui se plaignent du RSI, on a l'impression que c'est tout ce qui est auto-entrepreneurs et tout ça. Et il faut bien savoir que tout ce qui est auto-entrepreneurs, ils n'ont pas de cabinet comptable, c'est eux qui dialoguent directement avec le RSI. On n'a pas forcément les bons sons de cloche de la part de ces gens-là. »

(Franck O., 51 ans, électricien en EURL, un salarié)

Le légalisme en question se comprend dans le cadre de la trajectoire historique du groupe socioprofessionnel, dont les auto-entrepreneurs dans le bâtiment, ici évoqués, sont vus comme des avatars fâcheux. La mémoire collective du groupe, telle qu'elle s'est entendue chez des enquêtés au minimum quinquagénaires, reste empreinte d'un dispositif fiscal qui a durablement marqué la condition artisane en France, celui du forfait (1934-1999). Copié en 1934 sur la fiscalité agricole, le dispositif

«part du principe que la détermination précise des bénéfices suppose des moyens comptables trop contraignants pour des entrepreneurs isolés. Le bénéfice est alors estimé en commun par l'administration et l'artisan. En deçà d'un certain plafond, l'entrepreneur n'est pas imposé sur son bénéfice réel (réputé inconnu et inconnaissable avec précision) mais selon son bénéfice forfaitaire. À l'origine, il s'agit de simplifier les formalités. Les relations entre les artisans et l'administration sont réduites à la négociation, à intervalles espacés, de ce forfait mais la négociation est souvent âpre ${ }^{32} »$.

(PERRIN, 2007, p. 26)

Certains de nos enquêtés font un lien direct entre ce dispositif et la médiocrité des pensions de leurs prédécesseurs.

« À l'époque, les anciens, ils cotisaient sur la base d'un forfait, ils n'étaient pas au réel comme moi quand j'ai commencé, et ils payaient leurs impôts sur ce forfait. Donc, beaucoup d'artisans se débrouillaient pour ne pas dépasser le forfait, et l'argent, ils se le mettaient dans la poche. Sauf que quand ils sont arrivés à l'âge de la retraite, vu que le forfait était petit, ben leur retraite était petite. »

(Franck O., 51 ans, électricien en EURL, un salarié)

L'incitation fiscale à déclarer un revenu inférieur à un forfait et la pratique généralisée du travail non déclaré qui allait de pair forment la référence principale contre laquelle le profil de nos enquêtés se dresse, en nourrissant un sentiment légaliste qui se concrétise par l'acquittement de leurs cotisations sociales. Contrairement aux anciens, « négligents » et incités à l'être, ils estiment ne plus participer au far west socio-fiscal créé par le régime du forfait. Le régime dérogatoire de la microentreprise dans lequel a été fondu celui de l'auto-entreprenariat a perpétué en partie la logique du forfait pour

32. Nos entretiens évoquent des « foires d'empoigne » arbitrées par des commissions départementales. 
des artisans déclarant un chiffre d'affaires bas ${ }^{33}$. Aussi les indépendants classiques les assimilent-ils aux aînés tandis qu'eux-mêmes en forment le contrepoint marqué du sceau du sérieux dans le règlement de leurs charges : « Moi, on me demande de cotiser, je cotise. [...] Celui qui est habitué à payer [ses charges], il les paye. ${ }^{34}{ }{ }^{2}$

Comme le pointait un rapport écrit au début du processus d'alignement (1973) :

« Contrairement aux salariés, les petits indépendants doivent payer leurs cotisations sociales avec de l'argent qui est déjà entré dans leur caisse. Cette différence est plus importante qu'il n'y paraitt en première analyse. Il n'est pas sûr que les salariés de toutes classes et de toutes conditions accepteraient de payer de bonne grâce leurs cotisations sociales s'ils avaient eux-mêmes à le faire. Leur discipline apparente masque des divergences de point de vue que seule "l'automaticité du système" unifie. »

(LANCO, ZARCA, 1974, p. 134)

L'enquête permet de préciser que cette expérience spécifique des travailleurs non salariés par rapport aux salariés est aussi, et peut-être surtout, une expérience qui distingue les vrais indépendants de ceux qu'ils considèrent comme des faux indépendants. Le conformisme légal, autrement dit le fait que l'expert-comptable garantisse que les comptes renvoient, selon les termes consacrés, une image « vraisemblable et cohérente » de la réalité économique de l'homme-entreprise, constitue ainsi une frontière professionnelle. Alors que d'autres, anciens d'hier ou concurrents appréciés comme déloyaux d'aujourd'hui, étaient ou sont dispensés de la rigueur de ce réalisme, les artisans classiques s'y plient. Cette dimension est sans doute celle où la confiance entre l'expert-comptable et son client repose le plus sur leurs intérêts convergents à une comptabilité « professionnelle ». Pour autant, cette confiance présente des limites comme en témoigne la constitution autonome de « cagnottes », sinon concurrentes, du moins parallèles au « tiroir-caisse », dont la présentation officielle est confiée au cabinet comptable.

\section{Quand le sens de l'indépendance dans le métier conduit à relativiser les conseils de l'expert-comptable}

L'artisan a de nombreuses bonnes raisons de faire des pas de côté par rapport à l'emprise potentielle de son expert-comptable. La métaphore médicale trouve ici aussi sa pertinence. De même que l'expérience que le patient a de sa (bonne) santé peut diverger des avis et préconisations de son médecin de famille, le réalisme professionnel propre au métier artisanal peut s'opposer au réalisme comptable ${ }^{35}$. Hommes de terrain,

33. Compte tenu de l'importance des effectifs concernés, le régime du forfait a mis du temps à disparaître. Il a coexisté jusqu'à sa suppression en 1999 avec le régime fiscal de la microentreprise créé en 1991.

34. Gérald C., 62 ans, charpentier en EURL, solo.

35. L'extrait d'entretien suivant avec un expert-comptable, rapporté par Carlos RAMIREZ (2005, p. 377), illustre ce conflit de réalismes : « J'ai vécu des situations terribles où on me disait, non c'est pas possible, les comptes ne peuvent pas montrer ça, mon entreprise ne va pas si mal que ça, je ne peux pas déposer mon bilan, vous vous rendez compte qu'est-ce qu'ils vont devenir mes salariés et dans la région, on me connaît, j’ai bonne réputation. » Un autre exemple, 
les artisans du bâtiment savent qu'ils doivent leur longévité à leur réputation de bon professionnel. Or la satisfaction et la fidélisation de leurs clients ainsi que les relations avec les confrères ne sont pas toujours compatibles avec les préceptes prodigués comme étant ceux d'une saine gestion.

«Les comptables, c'est comme les banquiers, ils vous donnent que des conseils, mais il faut les appliquer après ! [Rires] Le comptable vous dit : "Faites de la marge, vendez un peu plus cher." Et puis le banquier vous dit : "Faites rentrer vos sous, faites-vous payer tout de suite, faites rentrer des acomptes." C'est le langage qu'on entend couramment, tout le temps. Mais bon, il y a des tas de paramètres pour faire rentrer les sous. [...] Vous avez des impératifs, soit vous êtes tributaire d'un autre artisan $[\ldots]$, soit vous avez vos propres imprévus. Et c'est vrai qu'en demandant des acomptes, vous êtes doublement lié en termes de délai d'exécution. Le client, il comprend encore moins pourquoi il doit attendre ! »

(Jean-Luc B., 65 ans, menuisier en entreprise individuelle, trois salariés)

Jean-Luc B. poursuit en expliquant pourquoi face à l'injonction de marger plus, la déontologie lui dicte de "vendre au juste prix ».

Si les conseils de l'expert-comptable sont ainsi relativisés, c'est aussi parce que l'estime réciproque, évoquée plus haut, entre les deux indépendants que sont l'artisan et son comptable est mâtinée d'un sens des distances sociales. Prestation intellectuelle (cabinet) en direction ici de travailleurs manuels (chantier), la relation entre l'expertcomptable et son client est aussi une confrontation entre deux univers de dispositions. Une forme symbolique de domination sociale se loge d'ailleurs dans le fait que, la plupart du temps, l'artisan n'a affaire qu'aux comptables salariés du cabinet. C'est seulement à l'occasion du bilan annuel ou de rendez-vous engageant une décision importante (comme celle de changer de statut juridique) que l'expert-comptable se libère en personne. De fait, des expressions de dédain, tenant à cette distance sociologique enchâssée dans une relation commerciale qui se veut de proximité, s'entendent, en off, des deux côtés. Ainsi des artisans : « Mon comptable, je trouve qu'il se la pète »; «Vous savez, un comptable, c'est relativement bête et discipliné ${ }^{-36}$.» Les railleries sont également légion dans le milieu des cabinets locaux où les petites mains se plaignent de « dossiers bordéliques », de factures « sentant la sciure et le sang ${ }^{37} »$ transmises de manière irrégulière. L'un des experts-comptables rencontrés est bien conscient qu'il y a un risque que ces « petits clients » perçoivent du mépris de la part de son cabinet, et il explique la manière dont il le désamorce : «Comme je dis à mes clients du bâtiment :

\footnotetext{
pas forcément conflictuel mais montrant cependant une autonomisation de l'indépendant, concerne la mise au point de son «propre tableau de bord », lorsque lui-même ou un proche possède des compétences de gestion.

36. Respectivement : Franck O., 51 ans, électricien en EURL, un salarié ; Bertrand S., 51 ans, maçon en SARL, dix salariés.

37. Cette expression empruntée à C. RAMIREZ est associée à la déconsidération marquant les propos des professionnels quand ils parlent de « comptabilité de boucherie » (ou de menuiserie).
} 
on fait ce qu' on peut pour s'alimenter dans la vie ! [Rires] Alors, ils rigolent parce que pour eux, ce que je fais, moi, c'est mieux que ce qu'ils font eux ${ }^{38}$. »

Mais le sens des distances sociales n'est pas, lui non plus, aussi univoque qu'il y paraît à première vue. Sur la question de l'opportunité de « se faire sa retraite », la confrontation entre les deux univers professionnels de dispositions offre un jeu de miroir instructif, cette fois à l' avantage des artisans.

Constat sociologique sans doute le plus important de l'enquête, les artisans établis rencontrés misent clairement sur la retraite pour consacrer le sens d'une vie (de travail) réussie. Il s'agit d'abord de rester indépendant jusqu'à cette échéance.

«J'ai un but, c'est d'y arriver, parce que je n'ai pas encore réussi... Tant que je ne serai pas en retraite, on ne sait pas ce qu'il peut se passer. Mon but, c'est d'y arriver. Quand je serai arrivé au bout de ma carrière, ben j'espère..., là je pourrai dire : j'ai réussi. »

(Orelian C., 55 ans, plaquiste en SARL, solo)

Selon un trait culturel propre aux métiers du bâtiment, la réussite passe par la capacité à investir dans la pierre, capacité qui, d'une certaine façon, détermine et motive la décision même de s'installer en tant qu'indépendant.

«-Quels étaient vos objectifs lorsque vous avez repris l'entreprise?

- L'objectif, c'était déjà de pouvoir bien travailler. Et puis justement, pouvoir faire deux-trois petits trucs pour moi, pour ma retraite, tout ça. Donc c'est pour ça que j'ai fait un local professionnel, j'ai fait une maison à louer... Améliorer un peu notre train de vie quoi. »

(Jean-Marie C., 56 ans, plâtrier-peintre en SARL, quatre salariés, trois revenus locatifs au montant supérieur à sa pension estimée à $1500 €$ )

Comme signalé auparavant, l'importance des revenus locatifs au regard de la pension par répartition a été une donnée régulièrement enregistrée par les entretiens, régularité dont la validité dépasse le nombre d'artisans enquêtés puisqu'eux-mêmes l'ont présentée comme un accomplissement normé signant la réussite dans le métier. Si les ressources monétaires tirées des investissements dans la pierre et de l'activité non déclarée peuvent être présentées comme des « cagnottes », c'est parce qu'elles représentent un usage de la professionnalité (savoir-faire, matériel, réseau) qui échappe au contrôle comptable du «tiroir-caisse ». Évidemment, dans ces investissements immobiliers, les artisans du bâtiment ajoutent à un apport financier leurs compétences pour « retaper » les biens acquis. Sans détailler la multiplicité des formes pratiques que prend cet art de retaper, il convient d'insister sur le sentiment de « ne jamais perdre de l'argent » qu'il procure.

«-Qu'est-ce que tu as fait toi pour préparer ta retraite?

38. Alain V., expert-comptable directeur de cabinet, huit collaborateurs. 
- Ben donc, la maison, là, à H., et puis l'autre dans le Midi. Donc investir dans le dur, moi c'était mon truc ! J'ai toujours dit qu'investir dans de la pierre, tu ne perds jamais d'argent. Et puis c'est vrai, quand tu fais quelque chose toi-même, tu ne perds jamais d'argent. »

(Alberto C., 59 ans, ancien associé d'une société de maçonnerie, devenu marchand de biens à 55 ans, anciennement propriétaire de quatre maisons et de deux appartements vendus à la date de l'enquête, un revenu locatif et une maison en attente de location)

« Investir dans la pierre, c'est toujours la chose qu'il faut faire, par rapport à n'importe quel placement financier. »

(Lucien B., 62 ans, maçon retraité anciennement en SARL, trois revenus locatifs au montant supérieur à sa pension de $1700 €$ )

Il y a une fierté évidente à pouvoir compter sur cette source d'enrichissement, accessible pour eux, et qui, de plus, les autorise à ne porter qu'une attention distraite aux préconisations de placements financiers (dont les contrats Madelin) formulées par les acteurs du marché de l'assurance. S'ils ne peuvent que les inviter à être « raisonnables » (en leur recommandant de diversifier leurs placements et en les alertant sur les risques associés au fait d'être bailleur quand on est à la retraite), les comptables ne manquent pas d'exprimer leur admiration, ce qui est aussi une façon de reconnaître que cette pratique d'auto-constitution patrimoniale leur échappe largement. Une pointe d'envie s'entend ainsi chez l'un d'entre eux :

« Et ils travaillent pour eux-mêmes. Voilà, ils se donnent des coups de main. Ils vont passer une semaine chez le copain lui faire son chauffage. En échange le copain va venir la semaine d'après faire le toit. Et puis ils se font du patrimoine immobilier comme ça. Et effectivement, dans ce cas-là, on se dit qu'ils sont plus malins que nous. Enfin ils sont malins, ils s'en donnent la peine aussi hein, c'est vrai qu'ils bossent. Ils se lèvent à 6 heures du matin, ils tapent dedans, donc moi ça me paraît plus juste objectivement...»

(Cyril D., expert-comptable directeur de cabinet, quatre collaborateurs)

Un autre expert-comptable prend l'exemple de l'établissement de son propre bilan pour souligner le « gain autorisé » représenté par les travaux de restauration pour soi :

« Par rapport à quelqu'un qui achète l'appartement tout fait, ça leur coûte beaucoup moins cher, ils font ça eux-mêmes, le week-end, ou quand ils sont gérants de société, ils ont le droit de travailler pour eux en dehors de la société. Cela constitue donc un revenu qui n'est jamais imposé. [...] L'artisan, il travaille dans son appartement, ce revenu-là n'est jamais imposé, et pourtant, il se retrouve pour la même valeur dans son bâtiment : quand il met 100000 [€] dans un appartement en travail et en matériaux, ces 100000 là, c'est avant charges sociales, avant impôt. De même que moi, quand je fais mon bilan, j'ai le même avantage. Je fais mon bilan bénévolement, donc je n'ai pas à le payer, donc j' ai un gain. »

(Alain V., expert-comptable directeur de cabinet, huit collaborateurs) 
Ce dernier extrait conduit à poser la question de la frontière du «bénévolat » avec le non-dit relatif au travail non déclaré, qui ne peut être avoué à son expert-comptable. Du travail pour soi, le week-end, avec l'aide de copains-collègues, au travail non facturé pour autrui produisant une valeur soustraite aux taxes et prélèvements obligatoires, les situations limites sont légion. L'entrée de la retraite sur la question du travail non déclaré a surtout confirmé, en permettant de la préciser, une frontière normative, interne au milieu et décisive pour les artisans s'y positionnant comme établis. Elle passe entre ceux qui abusent du travail non déclaré et minent la profession et ceux qui, légalistes, en font selon les règles communément admises, c'est-à-dire suffisamment peu pour ne pas s'attirer la suspicion de leurs experts-comptables. Cette seconde position est celle revendiquée par le profil majoritaire du corpus ( «On en passe forcément à gauche. Vu les taxes. Mais on est obligé d'être raisonnable $\left.{ }^{39} »\right)$.

Finalement, si certains enquêtés quinquagénaires ont spontanément mis en avant le travail non déclaré comme une ressource sûre qu'ils mobiliseraient si le niveau des pensions servies venait à se dégrader, voire si le système obligatoire venait à faire faillite («S'il n'y a plus de pension, on fera du black ! [Rires $\left.]^{40} »\right)$, d'autres, parfois les mêmes, déplorent la concurrence déloyale d'artisans retraités qui ne respectent pas la norme, par définition floue, d'une pratique tolérée à la marge :

« J'ai des collègues à la retraite qui continuent de travailler au black, alors ça, ça m'énerve. Parce que c'est un truc, on s'est battu tout au long de notre vie contre le black, parce que le black, il faut reconnaitre que ça nous coule, hein. Ce qui nous pénalise, c'est les gars, dont ces anciens artisans, qui font du boulot au noir. »

(Rodolphe J., 65 ans, électricien retraité anciennement en EI, solo)

D'un point de vue salario-centré, on peut sans doute toujours aujourd'hui souligner « la force des particularismes professionnels qui freinent l'harmonisation des comportements de retraite entre salariés et non-salariés » et constater que « la réforme des conditions de retraite des travailleurs indépendants se heurte à une certaine inertie » (Dubois-LAMBert, 1997, p. 4). A contrario, on peut considérer que ces «particularismes » professionnels sont et restent au principe d'une autre conception de la retraite que celle portée par les institutions nationales du salariat.

Cette conception alternative n'a rien de surprenant pour une approche économique qui prend en compte la double nature des revenus d'activité des indépendants (rémunération du travail et du capital) et aussi la porosité et la convertibilité de leurs actifs patrimoniaux : typiquement, l'atelier de l'artisan se trouve dans sa résidence principale ; plus subtilement, selon un montage préparant une transmission, l'acquisition personnelle d'un bâtiment professionnel permet de percevoir un loyer à la retraite.

39. Jacques R., 55 ans, maçon en SARL, trois salariés.

40. Franck O., 51 ans, électricien en EURL, un salarié. 
Quant à l'approche sociologique, elle peut documenter la manière dont les comportements optimisateurs prêtés à ces catégories de travailleurs opèrent et se perpétuent de manière située. Dans cette perspective, qui est aussi celle d'une sociologie dont l'apport à la connaissance des indépendants n'est pas « oblique » (CÉLÉRIER, 2016), ni a fortiori empreint de « la caricature habituelle de l'artisan poujadiste qui a vraisemblablement contribué à écarter les chercheurs de cette population » (PERRENOUD, 2008, p. 98), l'article s'est attaché, sur un profil circonscrit d'indépendant, à dégager deux mécanismes de mise au second plan de la pension issue du système de retraite par répartition.

L'attention à cet acteur discret mais clé de l'activité des petites entreprises qu'est l'expert-comptable a d'abord permis de comprendre comment le réalisme comptable a une portée socialisatrice pour l'indépendant. Elle se manifeste par une sensibilisation à la visibilité de «l'argent qui sort ». Conditionnée par le statut juridique d'exercice, la hiérarchisation comptable des cotisations révèle un rapport à la protection sociale obligatoire qui consiste à minimiser les versements d'argent perçus comme sans garantie de récupération. La préoccupation de ne pas perdre de l'argent est également au cœur du second mécanisme mis en évidence. Investir dans la pierre, la retaper pour se constituer un patrimoine immobilier de rapport, représente une manière spécifique aux artisans du bâtiment de créer une valeur qui, d'une certaine façon, est sanctuarisée. Mise en œuvre de leur art de faire pour leur propre usage, cet ajout de valeur est largement soustrait aux ponctions socio-fiscales. Démontrant la capacité qu'ont les professionnels du bâtiment de prendre leurs distances avec les conseillers en patrimoine, cette seconde logique prend les confrères comme références : il y a une façon particulière de tenir son rang d'établi dans le métier en usant ainsi de ses ressources professionnelles spécifiques pour se mettre à l'abri du besoin dans la perspective de ses vieux jours.

\section{BibLIOGRAPHIE}

Bessière C., De Paoli C., Gouraud B., Roger M. (2011), «Les agriculteurs et leur patrimoine : des indépendants comme les autres ? », Économie et statistique, $\mathrm{n}^{\circ} 444-445$, pp. 55-74.

BLANPAIN N. (2000), « Le patrimoine des indépendants diminue fortement lors du passage à la retraite $»$, Insee première, $\mathrm{n}^{\circ} 739$.

BonNeTÊTE F., Bignon N. (2017), « Les créations d'entreprise en 2016. La plus forte hausse depuis six ans, portée par les transports », Insee première, $\mathrm{n}^{\circ} 1631$.

BOURDIEU J., RAPOPORT B., Roger M. (2014), « Montant et composition du patrimoine des indépendants, avant et après le départ à la retraite », Économie et statistique, $\mathrm{n}^{\circ} 472-473$, pp. 169-193.

Bovigny A. (1995), La Gestion et la prévention fiscale des PME. Centres de gestion et association agréés, Paris, Presses universitaires de France. 
BRUNO A.-S. (2014), « Retour sur un siècle de protection sociale des travailleurs indépendants. France $\mathrm{Xx}^{\mathrm{e}}$ siècle » in Célérier S. (dir.), Le Travail indépendant. Statut, activités et santé, Rueil-Malmaison, Éditions Liaisons, pp. 31-52.

Bulteau S., Verdier F. (2015), Rapport au Premier ministre sur le fonctionnement du RSI dans sa relation avec les usagers, rapport gouvernemental, septembre.

CÉLÉRIER S. (2016), « Les apports obliques de la sociologie à l'étude des travailleurs indépendants », in Haut Conseil du financement de la protection sociale, La Protection sociale des non-salariés et son financement. Contributions des chercheurs et des administrations, tome 2 , pp. 5-20.

Cese (Conseil économique, social et environnemental), Weber M. (rapp.) (2015), « Le régime social des indépendants (RSI) », Les Avis du Conseil économique, social et environnemental, $\mathrm{n}^{\mathrm{O}} 2015-25$.

ChiAPEllo È., RAMIREZ C. (2004), « La sociologie de la comptabilité : une introduction », Comptabilité - Contrôle - Audit, tome 10, $\mathrm{n}^{\mathrm{O}} 3$, pp. 3-5.

COR (CONSEIL D' ORIENTATION DES RETRAITES) (2009), « Les régimes de retraite complémentaires du régime social des indépendants », Document de travail, $\mathrm{n}^{\circ} 9$.

Dietrich A., Moysan-Louazel A. (2012), « Conflits de territoire dans la profession d'expertcomptable », Revue française de socio-économie, $\mathrm{n}^{\circ}$ 9, pp. 227-245.

Dubois-LAmbert A. (1997), «Agriculteurs et indépendants face à la retraite », Inra sciences sociales, $\mathrm{n}^{\circ} 4$.

HCFi (HAUt CONSEIL DU FINANCEMENT DE LA PROTECTION SOCIALE) (2016), La Protection sociale des non-salariés et son financement, tome 1, septembre.

IGAS (INSPECTION GÉNÉRALE DES AFFAIRES SOCIALES), IGF (INSPECTION GÉNÉRALE DES FINANCES), (2016), Évolutions de l'assiette et des modalités de calcul et de recouvrement des cotisations sociales des travailleurs indépendants, rapport interministériel, juillet.

INSEE (2015), Emploi et revenus des indépendants, Paris, Insee ; en ligne : https://www.insee. fr/fr/statistiques/fichier/1374698/Revaind15.pdf, consulté le 13 juillet 2017.

INSEE (2016), Les Revenus et le patrimoine des ménages, Paris, Insee ; en ligne : https://www. insee.fr/fr/statistiques/fichier/2017614/REVPMEN16.pdf, consulté le 13 juillet 2017.

LANCO P., ZARCA B. (1974), Préparation financière de la retraite des petits indépendants, rapport à l'Organic et la Cancava.

LEROY M. (2003), « Sociologie du contribuable et évitement de l'impôt », European Journal of Sociology, vol. 44, n 2, pp. 213-244.

Malpot J.-J., MissèGue N. (1996), « Patrimoine des indépendants : une autre logique d'accumulation », Économie et statistique, n 296-297, pp. 91-112.

Perrenoud M. (2008), « Les artisans de la "gentrification rurale” : trois manières d'être maçon dans les Hautes Corbières », Sociétés contemporaines, $\mathrm{n}^{\circ}$ 71, pp. 95-115.

PERRIN C. (2007), Entre glorification et abandon. L'État et les artisans en France (1938-1970), Paris, Comité pour l'histoire économique et financière de la France. 
RAMiRez C. (2005), Contribution à une théorie des modèles professionnels. Le cas des comptables libéraux en France et au Royaume-Uni, thèse de doctorat en sociologie, Paris, École des hautes études en sciences sociales.

RsI (2015), L'Essentiel du RSI en chiffres, édition 2015 ; en ligne : https://www.rsi.fr/uploads/ tx_rsirss/Essentiel_du_RSI_en_chiffres_Version_integrale_Edition2015_donnees2014.pdf, consulté le 13 juillet 2017.

WanecQ T., Roux E., QueYranne M. (2008), Les Prélèvements obligatoires des indépendants, rapport, Paris, Conseil des prélèvements obligatoires. 